\title{
Modelagem da Dispersão de Poluentes Emitidos por Queimadas Usando a Técnica GILTT
}

\author{
Marcos A. Carraro* Daniela Buske Jonas C. Carvalho Regis S. Quadros \\ Programa de Pós-Graduação em Modelagem Matemática, PPGMMat, UFPel \\ 96010-900, Campus Capão do Leão, Capão do Leão, RS \\ E-mail: marcos.a.carraro@hotmail.com ; daniela.buske@ufpel.edu.br ; jonas.carvalho@ufpel.edu.br ; \\ regis.quadros@ufpel.edu.br
}

\begin{abstract}
RESUMO
Devido ao caráter turbulento do campo de vento na camada limite atmosférica, torna-se extremamente difícil o estudo da dispersão e do transporte de contaminantes na atmosfera. Por causa da complexidade da turbulência, o estudo da dispersão de poluentes na atmosfera emprega modernas metodologias disponíveis na comunidade científica. Neste aspecto, ferramentas teóricas e dados observacionais são combinadas com simulações numéricas com o objetivo de se compreender e de descrever, em uma maneira mais adequada, o fenômeno de transporte turbulento. As ferramentas teóricas são ainda hoje um desafio, uma vez que a dispersão de poluentes ocorre em um campo turbulento que possui um número infinito de graus de liberdade. Simplificações nas equações são obtidas e validadas por experimentos de campo que auxiliam na compreensão do processo de difusão. Os modelos matemáticos são um instrumento útil no entendimento dos fenômenos que controlam o transporte, a dispersão e a transformação físicoquímica dos poluentes na atmosfera.
\end{abstract}

$\mathrm{Na}$ estimativa do campo de concentração de poluentes na baixa atmosfera, emprega-se, normalmente, a equação de advecção-difusão, sendo que os modelos Eulerianos de dispersão têm como característica a solução desta. Na maioria dos casos encontrados na literatura, esta equação é resolvida analiticamente apenas em situações extremamente simplificadas, quase sempre com coeficientes de difusão constantes. Muitos avanços têm sido feitos no estudo da dispersão de poluentes utilizando o método conhecido na literatura como GILTT (Generalized Integral Laplace Transform Technique) para resolver a equação de advecção-difusão [4]. A idéia básica do método GILTT é a construção de um problema auxiliar de Sturm-Liouville associado ao problema estacionário, determinação da técnica da transformada integral em uma série truncada usando como base as autofunções do problema de Sturm-Liouville resolvido, substituição desta expansão no problema original. Tomando momentos, obtém-se uma equação diferencial ordinária matricial que é resolvida analiticamente pela técnica da transformada de Laplace e diagonalização.

O objetivo deste trabalho é investigar o problema de emissões de poluentes resultante de queimadas e o transporte transfronteiriço deste poluente. Especificamente, investigar um evento ocorrido no mês de agosto de 2010, conhecido como chuva laranja, onde a pluma de poluentes advinda das queimadas ocorridas no cerrado brasileiro foi transportada ate o Rio Grande do Sul provocando a chuva laranja no dia 22.08.2010. Para atingir este objetivo utiliza-se a técnica GILTT para resolver analiticamente a equação de advecção-difusão.

Neste trabalho, considera-se a equação de advecção- difusão com dependência temporal do campo de velocidades (por simplicidade, sem reações químicas), escrita como:

$$
\frac{\partial C}{\partial t}+u(z, t) \frac{\partial C}{\partial x}+v(z, t) \frac{\partial C}{\partial y}=\frac{\partial}{\partial z}\left(K_{z} \frac{\partial C}{\partial z}\right)
$$

sujeita a condições de contorno de fluxo nulo no solo e no topo da camada limite atmosférica, e condição de fonte contínua $C\left(0,0, H_{s}, t\right)=Q \delta\left(x-x_{0}\right) \delta\left(y-y_{0}\right) \delta\left(z-H_{s}\right)$ e condição inicial nula.

Utilizando a idéia do método da decomposição [1], considera-se que a concentração pode ser expandida na série truncada:

* Bolsista de Mestrado CAPES 


$$
C(x, y, z, t)=\sum_{n=0}^{N} C_{n}(x, y, z, t)
$$

Substituindo a série na equação (1) obteremos uma equação e $(n+1)$ funções incógnitas $\left\{C_{n}(x, y, z, t)\right\}$. Este procedimento nos leva a um conjunto recursivo de equações difusivas cuja solução é conhecida. Obviamente esta construção não é única. O conjunto recursivo escolhido é:

$$
\left\{\begin{array}{l}
\frac{\partial C_{0}}{\partial t}-\frac{\partial}{\partial z}\left(K_{z} \frac{\partial C_{0}}{\partial z}\right)=0 \\
\frac{\partial C_{n}}{\partial t}-\frac{\partial}{\partial z}\left(K_{z} \frac{\partial C_{n}}{\partial z}\right)=S_{n}(x, y, z, t)
\end{array}\right.
$$

onde $S_{n}(x, y, z, t)=-u \frac{\partial C_{n-1}}{\partial x}-v \frac{\partial C_{n-1}}{\partial y}$ para $n=1: N$. O primeiro problema do conjunto recursivo satisfaz a condição inicial, as condições de contorno e de fonte do problema (1), enquanto que os demais problemas satisfazem condições homogêneas.

Os dados micrometeorológicos específicos do local da queimada podem ser obtidos com os modelos de mesoescala BRAMS [3] e WRF [2]. O acoplamento do modelo GILTT com um modelo de mesoescala é mais realístico e, portanto adequado para o estudo do caso.

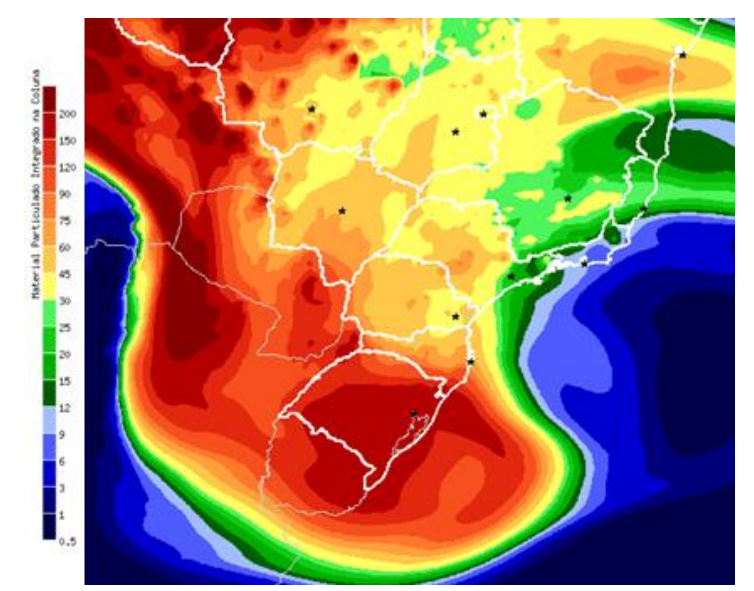

Figura 1: Simulação realizada com o modelo CATT-BRAMS de material particulado fino na coluna $\left(\mathrm{mg} / \mathrm{m}^{2}\right)$. Previsão para 18UTC 22.08.2010. Fonte: GMAI/INPE/CPTEC.

Palavras-chave: Queimadas, Solução Analítica, Modelagem da Dispersão de Poluentes

\section{Referências}

[1] G. Adomian, 1988. A Review of the Decomposition Method in Applied Mathematics, J. Math. Anal. Appl., Vol. 135, pp. 501-544, (1988).

[2] G.A. Grell,S.E. Peckham, R. Schmitz, S.A. Mckeen, J. Wilczak, B. Eder, Fully coupled "online" chemistry within the WRF model. Atmos. Environ., vol. 39, pp.6957-6975, (2005).

[3] K.M. Longo, S.R. Freitas, M. Pirre, V. Marecal, L.F. Rodrigues, J. Panetta, M.F. Alonso, N.E. Rosario, D.S. Moreira, M.S. Gacita, J. Arteta, R. Fonseca, R. Stockler, D.M. Katsurayama, A. Fazenda, M. Bela, M.. The chemistry CATT-BRAMS model (CCATT-BRAMS 4.5): a regional atmospheric model system for integrated air quality and weather forecasting and research. Geosci. Model Dev. Discuss., vol. 6, pp. 1173-1222, (2013).

[4] D.M. Moreira, M.T. Vilhena, D. Buske, T. TIRABASSI, The state-of-art of the GILTT method to simulate pollutant dispersion in the atmosphere, Atmos. Research, vol. 92, pp. 1-17, (2009). 